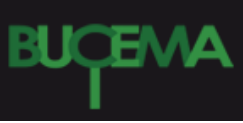

Bulletin du centre d'études médiévales d'Auxerre | BUCEMA

Hors-série $n^{\circ} 7 \mid 2013$

Les nouveaux horizons de l'ecclésiologie : du discours clérical à la science du social

\title{
Yves Congar et la sémantique du magistère
}

\section{Philippe Büttgen}

\section{(2) OpenEdition}

1 Journals

\section{Édition électronique}

URL : https://journals.openedition.org/cem/12870

DOI : $10.4000 /$ cem. 12870

ISSN : 1954-3093

Éditeur

Centre d'études médiévales Saint-Germain d'Auxerre

Référence électronique

Philippe Büttgen, «Yves Congar et la sémantique du magistère », Bulletin du centre d'études médiévales d'Auxerre | BUCEMA [En ligne], Hors-série n 7 | 2013, mis en ligne le 23 avril 2013, consulté le 04 mars 2023. URL : http://journals.openedition.org/cem/12870 ; DOI : https://doi.org/10.4000/cem.12870

Ce document a été généré automatiquement le 4 mars 2023

\section{(c) (i) (3) (2)}

Creative Commons - Attribution - Pas d'Utilisation Commerciale - Partage dans les Mêmes Conditions 4.0 International - CC BY-NC-SA 4.0

https://creativecommons.org/licenses/by-nc-sa/4.0/ 


\title{
Yves Congar et la sémantique du magistère
}

\author{
Philippe Büttgen
}

1 Il y a plusieurs portes pour entrer dans l'œuvre d'Yves Congar ${ }^{1}$. La première est la grande porte, celle que Congar a d'emblée ouverte, la porte d'une théologie de l'œcuménisme, ou si l'on veut parler rigoureusement: d'une fondation théologique de l'œcuménisme catholique. En ouvrant cette porte, on accédera aux grandes études que Congar a données en ecclésiologie et en pneumatologie, dans un parcours qui relie entre beaucoup d'autres points le Sainte Église de 1963 au Je crois en l'Esprit Saint de 1979-1980 2 . Hervé Legrand a bien montré que ces travaux ne prennent leur sens que rapprochés du coup d'envoi œcuménique de Congar, le Chrétiens désunis de $1937^{3}$.

2 Mais il existe encore d'autres portes, plus petites. Elles sont faites pour ceux qui préparent leur entrée dans Congar. La grandeur de Congar est d'avoir prévu ces petites portes, d'avoir pratiqué d'autres ouvertures, pour ceux que ni la discussion œcuménique, ni même l'histoire de la théologie catholique n'intéressent prioritairement.

3 Nous nous retrouvons aujourd'hui à quelques-uns, médiévistes, modernistes, historiens et philosophes, autour du projet d'une mise en mouvement de l'histoire par l'histoire de l'Église. De quoi s'agit-il ? Un préalable va consister à nous emparer de l'histoire des doctrines ecclésiologiques. On voudrait proposer un double regard, historien et ecclésiologique, une réflexivité partagée sur l'histoire de l'Église, de façon à ce que l'objet « Église » relance l'histoire et d'autres choses encore dans la réflexion : un sens de la norme doctrinale notamment, et de l'institution du vrai. Dès cette étape préliminaire nous rencontrons Congar, et avec lui une ecclésiologie d'essence historique dont le P. Legrand, de nouveau, a montré toute la singularité au regard des méthodes de l'histoire.

4 C'est là, il est vrai, un horizon autant qu'une ouverture. Il faut donc préciser. Je me propose d'effectuer sur un objet particulier la mise en mouvement de l'histoire par l'histoire de l'Église. Cet objet particulier, c'est la doctrine, qu'à quelques-uns nous travaillons à faire entrer dans l'agenda des historiens : la doctrine, dans sa réalité 
double et simultanée de forme de savoir, mode spécifique de rassemblement des énoncés vrais, et de norme de foi, capacité historiquement accordée à ce rassemblement du vrai, à sa transmission et à son enseignement d'agir sur les convictions et les comportements des hommes ${ }^{4}$.

S'agissant de Congar, et s'agissant de ce projet d'histoire du faire-doctrine médiéval et moderne, une précision s'impose encore. Congar est l'auteur de grandes études sur la notion de doctrine. Elles se trouvent notamment dans le recueil assemblé en 1984, Thomas d'Aquin. Sa vision de théologie et de l'Église, qui contient un "Tradition et 'Sacra doctrina' chez saint Thomas d'Aquin » de 1963, ainsi que l'article paru dans les Mélanges Chenu de 1967, "Le moment 'économique' et le moment 'ontologique' dans la Sacra Doctrina », sous-titré " (Révélation, théologie, Somme théologique) » ${ }^{5}$. Mais je ne sais rien de saint Thomas, et il y a aujourd'hui dans cette salle quelqu'un devant qui il n'est pas possible de feindre.

6 À une telle ignorance, Congar offre toutefois une heureuse échappatoire. Il est vrai que le rapport qu'il entretenait avec la tradition du savoir thomiste était plus oblique que chez d'autres théologiens dominicains. On apprend donc beaucoup de l'histoire de la doctrina, de ses problèmes aussi, dans plusieurs études de Congar, en particulier dans celles qu'il a consacrées à la notion de magistère. Au terme de ce préambule, j'en arrive à deux articles de Congar, parus l'un et l'autre en 1976 dans la Revue des Sciences Philosophiques et Théologiques. Le premier s'intitule «Pour une histoire sémantique du terme 'magisterium' »; le second propose un « Bref historique des formes du 'Magistère' et de ses relations avec les docteurs ». De ces deux textes, je voudrai proposer une lecture orientée vers les deux intersections d'une l'histoire de l'ecclésiologie avec l'histoire conceptuelle de la doctrina, et d'une histoire de la doctrine avec celle du magistère.

7 Cette lecture procédera par réductions successives. J'examinerai premièrement le lien entre les deux articles et ce qu'il donne à comprendre des intentions de Congar; deuxièmement l'enchaînement des séquences à l'intérieur du premier article, sur la sémantique de magisterium; troisièmement enfin, l'enchaînement des significations originaires de magisterium au commencement de ce premier article. Cet examen permettra de poser à Congar de nouvelles questions, celles que semble désormais requérir une histoire intégrée de l'Église, du magistère et de la doctrine.

8 Nous avons affaire à un dispositif éditorial assez remarquable : deux articles de Congar pour la Revue des sciences, parus la même année, dans le même volume, à la suite l'un de l'autre ${ }^{6}$. Pourquoi alors deux articles?

9 Lisons les incipits. Premier article, "Pour une histoire sémantique du terme 'magisterium'»; Congar dit: "Il s'agit seulement du mot». Second article, "Bref historique des formes du 'Magistère' et de ses relations avec les docteurs »: « C'est une histoire qui n'a jamais été faite dans son ensemble». Nous sommes en 1976 : Congar, à 72 ans, a l'impression d'aborder un continent nouveau. Il l'abordera par un "mot", magisterium. Le lien entre les deux articles se découvre à l'intuition; à la réflexion, il est plus difficile à cerner. Le premier article entame une étude sémantique, en reconnaissant que celle-ci ne peut qu'être partielle au regard d'une question plus générale («il s'agit seulement du mot»). Mais quelle est cette question d'ensemble ? Le deuxième article, à son tour, semble ne traiter qu'un aspect particulier, le magistère et les docteurs, même si, comme dit Congar, cet aspect n'a jamais été traité «dans son ensemble ». 
10 Le second article inclut en son centre un résumé du premier. De ce résumé doit se déduire l'objectif commun :

Résumons ici en quelques mots ce que la Note qui précède celle-ci établit clairement. Le mot « magisterium » ne désigne pas alors [i.e. au Moyen Âge] ce que nous appelons «le Magistère ». Chez les Pères, au Moyen Âge et jusqu'aux années 20-30 du XIX $\mathrm{X}^{\mathrm{e}}$ sì̀cle, magisterium signifie simplement la situation, la fonction ou l'activité de quelqu'un qui est en position de magister, c'est-à-dire d'autorité dans un domaine particulier. (...) L'activité pouvait être celle d'enseignement. Dans ce cas, magisterium rejoignait matériellement le sens moderne de "magistère ", mais jamais avant le $\mathrm{xIX}^{\mathrm{e}}$ siècle, il ne signifie exactement ce que nous appelons «le magistère » (103, italiques de l'auteur dans tout ce qui suit, sauf mention contraire).

11 C'est ici le relais entre les deux articles, le cœur du propos. Et pourtant Congar ne peut s'exprimer que d'une manière malaisée, répétitive : nous sommes invités à penser les relations entre "magisterium ", "magistère » et «le Magistère ", sans autre outil que notre compréhension intuitive de ce que l'article défini, "le Magistère», et éventuellement l'emploi de la majuscule peuvent produire de modification dans le sens du mot.

On reviendra sur ce point dans la partie suivante, pour décrire le cheminement du premier texte de Congar. Interrogeons-nous ici sur le lien entre la thèse résumée ici - le " magistère » n'a pas toujours été " le Magistère » - et le propos qui entoure cette thèse dans le second article. Il s'agit de penser la place d'une population particulière, celle des docteurs, parmi tous ceux auxquels les auteurs d'Église reconnaissent la possession d'un magisterium. Congar fait mention, "dès la seconde moitié du $\mathrm{II}^{\mathrm{e}}$ siècle ", d'« une certaine tension entre la spéculation des docteurs et le témoignage apostolique lié à la succession des ministres ordonnés" (100). Cette tension est celle de deux « enseignements »: « enseignement de type doctoral-scientifique et enseignement de type pastoral ", magisterium cathedrae pastoralis, ou pontificalis, et magisterium cathedrae magistralis, selon une distinction de saint Thomas (In IV. Sent., dist.19) qui est mentionnée dans les deux articles (103, cf. 92). Disons encore, en simplifiant, catéchèse et théologie. Cette distinction pose le problème de la création théologique et de sa place dans l'enseignement de l'Église ; ou encore de la part respective de la proclamation et de la spéculation dans le Magistère.

Congar observe ici des variations. Tantôt les docteurs se sont tenus à côté des évêques et des papes, jusqu'à revendiquer des droits supérieurs aux leurs (conciles des XIV $\mathrm{xv}^{\mathrm{e}}$ siècles); tantôt ce sont les évêques et les papes eux-mêmes qui se sont faits théologiens. Ainsi au Xxe siècle :

Les encycliques de Léon XIII et de Pie XII font de la théologie. Elles ne sont pas purement expression du témoignage apostolique selon les besoins du temps, mais une doctrina de «cathedra magistralis » assumant des données de droit naturel, de sagesse humaine, de théologie classique (105).

La doctrina apparaît ici pour la première fois. Mais l'essentiel tient pour le moment dans ce constat: derrière les variations dont on parlait demeure, jamais dissipée, l'indistinction du pastoral et du théologique.

C'est là, pour Congar, un constat historique. Il admet plusieurs usages. Il explique, premièrement, un certain nombre d'évolutions que l'auteur juge dommageables, à commencer par la confusion, dans le «magistère papal», entre l'autorité et le "jugement dirimant un débat» (106). Cette évolution résulte d'un brouillage, par le 
savoir théologique, de la «fonction de prédication et de témoignage ». De ce point de vue explicatif, la spéculation endosse une responsabilité négative.

Dans le même temps, le constat historique fonde pour Congar une norme théologique. Le second texte de la RSPT formule en effet des recommandations claires. Il livre un plaidoyer pour la liberté de la recherche théologique, plaidoyer soutenu par l'exemple de Vatican II, illustration « assez éclatante » de ce que peut apporter la « collaboration de théologiens et des Pères conciliaires » (110). Notons la justification que se donne cet éloge d'une "théologie active, menée dans un climat religieux, fraternellement confiant et communiquant » (111). La défense de la liberté des docteurs se fait au nom de la nécessité de «ne pas séparer la forme du ministère apostolique du contenu de la Tradition " (108). Elle se réclame par conséquent de l'indistinction entre pastorat et théologie, entre proclamation et doctrine, dont Congar déplore par ailleurs les conséquences fâcheuses.

On l'aura compris: l'ecclésiologie historique de Congar, telle qu'illustrée par son approche du magistère, est aussi un art d'écrire. Les protestations de modestie de l'historien, parti d'une « note » lexicographique (« il s'agit seulement du mot »), mènent à une revendication théologique de grande ampleur. Les développements de Congar sur la première sémantique du magisterium appellent donc une lecture rétrospective. La clé de lecture se trouve dans les lignes finales:

S'il est permis de conclure un exposé historique sur une perspective théologique, nous dirons : le rapport entre docteurs et magistère appelle reconsidération. Cela suppose qu'on précise d'abord le statut du «magistère » dans l'Église, qu'on ne l'isole pas de la réalité vivante de l'Église (112).

L'art d'écrire congarien est un art du détail. Une étude en apparence particulière, celle de la relation entre docteurs et magistère, ouvre à un questionnement de l'Église et de sa " réalité vivante » à l'époque de Congar, il y a trente-cinq ans pour nous. Le procédé rétrospectif imposé par les deux textes de la Revue des Sciences va maintenant permettre de voir que toute la charge de ce questionnement est déjà contenue dans la première étude, sur la sémantique de magisterium.

Parcourons d'une phrase l'arc sémantique tracé pour magisterium, après avoir précisé que Congar extrait le mot d'un ensemble qui devrait aussi comprendre, dit-il, des termes comme cathedra, potestas ou auctoritas (85). Du latin classique à celui des Écoles médiévales et modernes, magisterium a successivement désigné une "situation d'autorité » (86), un enseignement, la doctrine résultant de cet enseignement et enfin le corps enseignant détenant l'autorité doctrinale. C'est à propos de ce dernier que Congar soigne le plus ses définitions : le "corps hiérarchique des pasteurs » (97), ou encore le "corps désignable de chefs hiérarchiques ayant autorité pour enseigner " (98), le « corps hiérarchique désignable» (94), constitue "le Magistère » avec article accentué, dans son « acception aujourd'hui courante » ou dans le « sens moderne » du terme $(86,91)$. À plusieurs reprises Congar insiste sur le fait que cette acception est tardive, et qu'elle ne se trouve véritablement ni dans le latin des Pères, ni dans celui des docteurs scolastiques. C'est la thèse que résume le deuxième article : "jamais avant le $\mathrm{XIX}^{\mathrm{e}}$ siècle, [magisterium] ne signifie exactement ce que nous appelons "le magistère" ". Il faut à présent apporter plusieurs précisions.

20 Première précision, documentaire : dans l'évolution sémantique qui mène au «sens moderne », comme dit Congar, du Magistère, une grande responsabilité est reconnue à des canonistes allemands du XIX ${ }^{e}$ siècle, Brendel, Walter, Philipps, qui, en se réclamant 
de la «doctrine catholique» (Brendel cité par Congar), ont identifié l'Église au «magistère (Lehramt), [à] l'Église enseignante (lehrende Kirche)» (94). En filigrane se dégage le contre-modèle de l'école historique de Tübingen et singulièrement de Johann Adam Möhler, que Congar avait publié dès 1938 dans la collection Unam Sanctam ${ }^{7}$. De 1938 à 1976, l'exemple de l'érudition ecclésiastique allemande aura constitué pour Congar une constante théologique.

21 Deuxième précision, argumentative : la démonstration de Congar est essentiellement traditionnelle. Derrière le Magistère dans son acception tardive de " corps hiérarchique désignable " (94), Congar recherche un sens originel et authentique. On comprend mieux, rétrospectivement, l'éloge de la "tradition active ", du "magistère vivant " dans le second article (107), et plus encore la manière qu'a Congar de définir ce magistère vivant comme «contenu de la Tradition» inséparable de la «forme du ministère apostolique " (108). Cette définition de la tradition comme contenu objectivé fait du magistère la conséquence de la tradition, et non l'inverse. Le magistère n'est pas, comme magistère, gardien de la tradition. Il ne prend ce rôle que si, « des deux activités classiques du magistère, conserver et définir ", c'est la conservation qui l'emporte sur la définition dogmatique. Pour atteindre ce but, il convient de comprendre la conservation comme acte de «témoigner de ce qu'on a reçu» (108). Congar loue le Concile Vatican II d'avoir « rétabli le rapport traditionnel de subordination de l'autorité au donné ou à l'objet, bref la primauté du quod sur le quo» (111). On notera avec intérêt que cette formulation a été permise par l'examen de deux définitions de la Tradition et de la regula fidei, l'une empruntée au luthérien Bengt Hägglund, l'autre au jeune Joseph Ratzinger : toujours le tropisme germanique de Congar (101) ${ }^{8}$.

Résumons: la critique par Congar des évolutions du Magistère se comprend comme traditionnelle, au nom d'une tradition objectivée et pensée, à ce titre, d'une façon littéralement conservatrice. Celui qui aura le mieux saisi l'esprit de cette critique est sans doute Henry Chadwick, dans les "Quelques réflexions sur le magisterium dans l'Église » qu'il offrait à Congar pour ses Mélanges de $1974^{9}$. Après avoir résumé l'opinion des Pères (Irénée) en affirmant que "le devoir du bon théologien » est de "maintenir ce qui est donné » ${ }^{10}$, il insiste sur les possibilités qu'offrait pour eux "l'acceptation de la tradition comme norme d'interprétation de l'Écriture ». À la suite, il caractérise une telle acceptation comme " une certaine forme de libération du passé, quand le texte écrit semble à la fois gênant et insuffisant " ${ }^{11}$. Quand on lit les propos de Congar sur l'objectivité de la Tradition, sur la nécessité de sa conservation et, tout aussi bien, de la libre discussion des théologiens, on comprend mieux de quoi il retourne essentiellement dans les deux articles de 1976 sur le magistère : ni plus ni moins que d'une libération de la Tradition.

Troisième et dernière précision, en forme cette fois d'interrogation. À la fin du premier article, Congar formalise l'évolution qu'il a retracée en opposant, dans magisterium, un "sens d'office et d'activité d'enseignement" au sens déjà vu de "corps hiérarchique des docteurs » (96-97). C'est, si l'on veut, la fonction contre la substance, à ceci près que la substance dont il s'agit ne peut pas être, pour les raisons que nous avons vues, celle du contenu de l'enseignement, mais bien celle du corps qui enseigne. Congar examine ici divers textes de Pie IX, Léon XIII et Pie XII. La même évolution apparaissait en filigrane $\mathrm{au}$ début du texte, quand la «fonction officielle et même hiérarchique d'enseignement " (sens patristique et médiéval de magisterium) était opposée au " corps des pasteurs ayant autorité pour exercer cette fonction » (sens contemporain [86]). 
24 Dans leur précision même, les formulations de Congar trahissent toutefois une difficulté : du strict point de vue terminologique, quel est le problème posé par l'acception contemporaine du Magistère ? Est-ce parce qu'il s'agit de «hiérarchie »? Apparemment non, puisque l'ancienne «fonction» d'enseignement opposée au moderne «corps » enseignant est elle-même « hiérarchique » ("fonction officielle et même hiérarchique d'enseignement ", insiste Congar [86]). Quelle différence y a-t-il, aux yeux de Congar, entre corps et hiérarchie? Un corps sans hiérarchie se conçoit peut-être, mais qu'est-ce qu'une hiérarchie sans corps? C'est au travers de telles questions, avec toute la part d'implicite à laquelle elles semblent s'affronter, que Congar se révèle grand théologien catholique.

La difficulté peut encore se formuler autrement. Au moment où il oppose entre elles deux acceptions du magistère, comme fonction d'enseignement et comme corps enseignant, Congar voit ces deux acceptions coexister dans les textes mêmes qu'il critique chez Pie IX et Pie XII. Louons à cette occasion l'extraordinaire oreille latine qui peut, dans un même texte, faire le départ entre ces deux significations, à quelques phrases de distance. Le même constat vaut pour l'évolution que cherche à retracer le premier article sur la sémantique de magisterium. À aucun moment Congar n'invoque une déviation du sens originel, une bifurcation ou un déraillement, du magistèrefonction au magistère-corps ou substance. Au contraire, il insiste sur l'inéluctabilité sémantique qui semblait devoir mener magisterium à son acception contemporaine. Ainsi à propos de Bernard de Fontcaude dès 1185 (91), puis d'olivi, de Guy Terreni et plus tard de Claude Lejay au Concile de Trente: "Avec les mots traditionnels, on approche [...] de l'usage moderne » (93). Ou encore, à propos d'un passage de Martin Gerbert, abbé de Saint-Blaise (mort en 1793) : «ce n'est pas encore exactement ce que nous appelons aujourd'hui "le Magistère", c'est-à-dire un corps hiérarchique désignable [...], mais on est bien proche de ce sens» (94). Immédiatement à la suite, Congar en arrive aux canonistes allemands qui distinguent la potestas magisterii de la potestas ministerii et de la potestas iurisdictionis. Ici une rupture est certes marquée, mais ce qui précède oblige à demander : s'agit-il d'une rupture, ou n'y a-t-il pas eu plutôt variation infinitésimale, clinamen voire nécessité sémantique? Et dans ce cas, à quelle autre nécessité renvoie celle du lexique?

26 Je voudrais clore cet exposé par une nouvelle série de questions. Celle-ci va viser la première séquence du premier article, qui examine l'évolution sémantique de magisterium dans le latin classique et le latin des Pères, avant même la problématique rupture dont il vient d'être question. «Magisterium a d'abord, dit Congar, le sens de position et autorité de qui est chef, magister» (textes de Lactance, d'Eusthate, de l'Ambrosiaster, 87); suit le "rôle d'enseignement " (textes d'Augustin, à propos du Maître intérieur, et de Grégoire le Grand, 89-90) ; on en arrive finalement au « contenu de l'enseignement, une doctrine » (textes de Cyprien et de Rupert de Deutz, 92).

Ici il faut continuer de citer Congar, et plus exactement la manière dont est présenté chez lui le passage d'un sens à l'autre. Soit successivement :

"Il était normal que, désignant d'abord une situation d'autorité ou de direction, magisterium ait été appliqué à un rôle d'enseignement » (89).

«Si magisterium s'appliquait particulièrement à l'enseignement, il était normal que le mot fût pris au sens objectif et désignât le contenu de l'enseignement, une doctrine » (92). 
Enfin à la même page, en forme de synthèse et pour déjà introduire à la signification contemporaine du Magistère :

«Si magisterium exprime une position d'autorité et si le terme a connu très tôt une application à l'ordre de la doctrine, il est normal qu'on rencontre des textes dans lesquels semble bien s'annoncer l'acception moderne du terme » (j. s.).

La sémantique du magistère ne connaît pas les coups de théâtre. Tout y est préparé ou, comme dit Congar, «normal ». Derrière ce «normal » réitéré se cache néanmoins une forêt de questions.

- Sur la normalité présumée du passage du pouvoir à l'enseignement: est-il si «normal» qu'une autorité se donne pour enseignante? N'est-ce pas au contraire l'une des propriétés les plus étranges et les plus enfouies du pouvoir en christianisme que de s'autoriser pour une si grande part des leçons qu'il délivre ? L'Église est une agence de salut à l'intérieur de laquelle toutes les opérations passent par une transmission de savoir. C'est un édifice de connaissance, tout autre chose par conséquent que l'implication simple d'une gnose : si vous savez, vous serez sauvé. Il est frappant qu'au moment d'affirmer la normalité de la transition que nous examinons, Congar allègue des textes dont lui-même reconnaît la difficulté. Ainsi le pape Célestin Ier à propos de saint Cyrille et de Nestorius au concile d'Éphèse: "porrexit dexteram magisterii sui». "Comment traduire?», demande Congar. Suit sous sa plume une proposition assez étourdissante, assortie d'un commentaire perplexe. Pour " porrexit dexteram magisterii sui », on pourrait avoir :

«'il lui a tendu sa main d'évêque-docteur'. Tel est, croyons-nous, le sens. 'Magistère' ne serait pas faux, mais serait sans doute trop précis, le mot ayant pris, nous le verrons, un sens plus défini » (90).

31 Par delà donc les affirmations de normalité, si nous ouvrons le mot magisterium, nous y trouverons une étrange fonctionnaire, "l'évêque-docteur ». Cette seule phrase d'un pape des premiers temps indique le lien entre les deux articles de Congar. Elle résume également le parcours qui mène de la sémantique en apparence modeste de magisterium à l'examen des «formes du 'magistère' » et de ses " relations avec les docteurs ». Dans cet examen tout aussi circonscrit en apparence, nous avons vu que Congar concentrait sa revendication ecclésiale, quinze ans après un Concile qui fut son concile.

Le texte que Congar mentionne à la suite de celui de Célestin Ier n'est pas moins fascinant. On lit dans l'homélie 70 de Maxime de Turin († 465), citée par Congar :

Et licet in Petro fides emineat, in Paulo doctrina praecellet, et magisterium tamen Pauli fidei plenitudo est, et credulitas Petri doctrinae est fundamentum (cit. 90).

Je tente, difficilement à mon tour, une traduction :

Et alors qu'en Pierre la foi l'emporte, chez Paul c'est la doctrine qui passe au premier plan. Pourtant c'est le magistère de Paul qui est plénitude de foi, la croyance de Pierre étant fondement de doctrine.

Congar commente: "où doctrina signifie enseignement au sens actif» (90). Dans l'étonnant chiasme des deux apôtres, de leur « doctrine » et de leur « foi », le magistère a rejoint la doctrina. Au passage, la sémantique de magisterium aura croisé ce qui a été pour elle, sinon son modèle - comment l'établir ? -, du moins un prédécesseur. Pour l'une des premières livraisons du Bulletin Du Cange, Henri-Irénée Marrou donnait en 1934 une étude sur " 'Doctrina' et 'disciplina' dans la langue des Pères de l'Église ", prémisse lexicale de son travail sur l'éducation dans l'Antiquité ${ }^{12}$. Dans cette rencontre bibliographique entre deux maîtres de l'érudition catholique française au $\mathrm{xx}^{\mathrm{e}}$ siècle, on 
pourra repérer le point de croisement entre doctrine et magistère dont j'étais parti au début de cet exposé.

- Sur la normalité présumée du passage de l'enseignement à la doctrine. Là encore on ne peut que questionner. Marrou rappelle que doctrina veut d'abord dire l'enseignement, envisagé comme action et comme contenu. Mais Congar parle bien de l'« ordre de la doctrine » au moment où, pour une troisième fois, il recourt à l'épithète "normal » pour qualifier l'évolution de magisterium vers ce sens contemporain qu'il conteste par ailleurs. Par la suite il sera question $\mathrm{d}^{\prime}$ ' une forme de doctrina analytique, questionnante, cherchant les rationes, mettant en œuvre des ressources proprement philosophiques, non seulement de raisonnement mais de contenu et de pensée » (103). L'«ordre de la doctrine»: tout enseignement doit-il être rassemblé en un corps d'énoncés doctrinaux ? Nous savons par expérience que non. Et pourtant : n'est-ce pas à nouveau l'un des traits les plus profonds du christianisme que la nécessité avec laquelle, chez lui, l'enseignement de vérité s'ordonne en corps de doctrine?

Sur cet écho qu'on pourra percevoir entre le corps de doctrine et le «corps hiérarchique de pasteurs » désigné par Congar se clôt la lecture que je voulais proposer des deux articles de 1976. Aux questions que soulève l'argumentation subtile de Congar au service d'une libération de la tradition se sont enchainées celles auxquelles nous sommes nous-mêmes confrontés aujourd'hui dans notre histoire de l'ecclésiologie: celle de l'enseignement comme pouvoir de vérité, celle du vrai comme doctrine. Norme de croyance et forme du vrai, disais-je en commençant : on aura compris que lorsque Congar parle de "normalité", il nous mène en vérité jusqu'aux couches les plus profondes d'un savoir en Église, c'est-à-dire tout de même à l'une des deux ou trois formes marquantes du savoir en Occident.

\section{NOTES}

1. Le caractère oral de l'exposé a été conservé pour cette publication intermédiaire.

2. Y. CONGAR, Sainte Église. Études et approches ecclésiologiques, Paris, 1963 (Unam Sanctam, 41) ; Je crois en l'Esprit saint, Paris, 2 vol., 1979-1980.

3. ID., Chrétiens désunis. Principes d'un « œcuménisme » catholique, Paris, 1937 (Unam Sanctam, 1), avec plus tard les bilans tirés dans les articles des Écrits réformateurs, J.-P. JossuA (éd.), Paris, 1995, (Textes en main), p. 255-348. Voir H. LEGRAND, "Yves Congar (1904-1995). Une passion pour l'unité », in Nouvelle Revue Théologique 126 (2004), p. 529-554.

4. Voir Ph. bÜtTGen, R. IMBACH, U. J. SCHNEIDER et H.J. SELDERhuis (dir.), Vera doctrina. Zur Begriffsgeschichte der Lehre von Augustinus bis Descartes. L'idée de doctrine de saint Augustin à Descartes, Wiesbaden, Otto Harrassowitz (Wolfenbütteler Forschungen, 123), 2009 et Ph. B., Th. KAUfMANN, U. J. SCHNEIDER et H. J. SELDERHUIS (dir.), Sacra doctrina. Lehre, Konfession und Gesellschaft in der Frühen Neuzeit. Doctrine, confession et société, XVI ${ }^{e}$-XVIII ${ }^{e}$ siècle, même éditeur, 2011 (sous presse).

5. Voir Y. CONGAR, Thomas d'Aquin. Sa vision de théologie et de l'Église, London, Variorum (Collected Studies Series, 190), 1984, textes II et XIII (la réimpression a conservé les paginations d'origine). 
6. Y. CONGAR, «Pour une histoire sémantique du terme 'magisterium' » et « Bref historique des formes du 'magistère' et de ses relations avec les docteurs", Revue des sciences philosophiques et théologiques 60 (1976), p. 85-98 et 99-112 (références dans le corps du texte, entre parenthèses). Ces articles ont été repris par deux fois dans les recueils Droit ancien et structures ecclésiales, London, Variorum, 1982 (Collected Studies Series, 159) et Église et papauté. Regards historiques, Paris, 1994 (Cogitatio fidei, 184). L'un des deux textes est paru en traduction anglaise : «A Brief History of the Forms of the Magisterium and Its Relations with Scholars ", in Ch. E. cURRAN et R. A. мсCоRміск (éd.), Readings in Moral Theology. The Magisterium and Morality, New York, 1982.

7. J.-A. [Johann Adam] MÖHLER, L'Unité dans l'Église ou le principe du catholicisme d'après l'esprit des Pères des trois premiers siècles de l'Église, tr. fr. A. DE LILIENFELD, Paris, (Unam Sanctam, 2), 1938 (rééd. coll. «Foi vivante», 1980). En français, voir récemment M. DENEKEN, Johann Adam Möhler, Paris, 2007 (Initiation aux théologiens). L'intérêt de Congar pour Möhler date du milieu des années trente et procède significativement de sa réflexion œcuménique : voir ses deux articles, «La pensée de Möhler et l'ecclésiologie orthodoxe», in Irénikon 12 (1935), p. 321-329 et « La signification œcuménique de l'œuvre de Möhler», in Irénikon 15 (1938), p. 113-130 (cf. E. LANNE, «Le Père Congar et la revue 'Irénikon' ", in A. VAuchez (dir.), Cardinal Yves Congar (1904-1995), Paris, 1996, p. 105-115 (Histoire).

8. La somme « conciliaire » de Congar, La Tradition et les traditions, 2 vol., Paris, Fayard, 1960-1963, a été réimprimée en 2010 aux Éditions du Cerf. Les références de Congar sont ici : J. RATZINGER, "Primat, Episkopat und Successio apostolica », in K. RAHNER et J. R. (dir.), Episkopat und Primat, Freiburg i. B. etc., Herder (Quaestiones disputatae, 11), $1961\left(1963^{2}\right)$, p. 37-59 ; B. HÄGGLUND, « Die Bedeutung der "regula fidei" als Grundlage theologischer Aussagen ", Studia theologica 12 (1958), p. 1-44. Bengt Hägglund (né en 1920), professeur d'histoire des dogmes à Lund, est aussi l'auteur d'un ouvrage en son temps important sur les sources médiévales de Luther: Theologie und Philosophie bei Luther und in der occamistischen Tradition. Luthers Stellung zur Theorie von der doppelten Wahrheit, Lund, Gleerup, 1955. Le Congar de Martin Luther, sa foi, sa réforme (Paris, [Cogitatio fidei, 119], 1983), l'aura connu par là.

9. H. CHADWICK, «Quelques réflexion sur le magisterium dans l'Église », in Le Service théologique dans l'Église. Mélanges offerts au Père Yves Congar pour ses soixante-dix ans, Paris, , 1974, p. 163-175 (Cogitatio fidei, 76), repris in Heresy and Orthodoxy in the Early Church, London, Variorum, 1991 (Collected Studies Series, 342).

10. Ibid., p. 166.

11. Ibid., p. 168 (j. s.).

12. H.-I. MARRou, "'Doctrina' et 'disciplina' dans la langue des Pères de l'Église », Archivum Latinitatis Medii Ævi 9 (1934), p. 5-25. Voir Histoire de l'éducation dans l'Antiquité, 2 vol., Paris, $1981^{6}$. L'étude de Marrou précède de peu une « Note de lexicographie philosophique médiévale » que Marie-Dominique Chenu a consacrée à «Disciplina » (RSPT 25 [1936], p. 686-692). En 1976, les deux articles de Congar prolongeaient la ligne «lexicographique » de l'École du Saulchoir, qui avait déjà abordé les questions traitées ici : de Chenu toujours, voir les deux études «'Authentica' et 'Magistralia'. Deux lieux théologiques aux XI'-XII ${ }^{\mathrm{e}}$ siècles ", Divus Thomas 28 (1925), p. 257-285 et "'Maître' Thomas est-il une 'autorité'? Note sur deux lieux théologiques au XII siècle: les 'auctoritates' et les ‘magistralia' », in Revue thomiste 30 (1925), p. 187-194. 
INDEX

Mots-clés : magistère, doctrine, docteur, enseignement, autorité, pasteur

\section{AUTEUR}

\section{PHILIPPE BÜTTGEN}

Université Paris 1 Panthéon-Sorbonne 